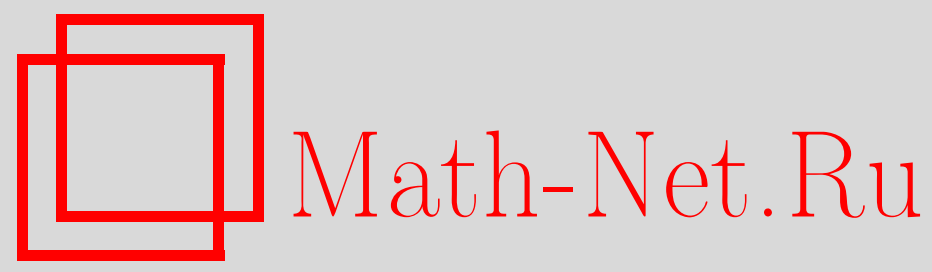

А. В. Маркелова, Дискретное логарифмирование в произвольных факторкольцах многочленов от одной переменной над конечным полем, Дискрет. матем., 2010, том 22, выпуск 2, 120-132

DOI: https://doi.org/10.4213/dm1099

Использование Общероссийского математического портала Math-Net.Ru подразумевает, что вы прочитали и согласны с пользовательским соглашением http://www . mathnet.ru/rus/agreement

Параметры загрузки:

IP : 34.229 .108 .108

26 апреля 2023 г., 16:01:57 


\title{
Дискретное логарифмирование в произвольных факторкольцах многочленов от одной переменной над конечным полем
}

\author{
() 2010 г. А. В. Маркелова
}

В статье рассматривается вопрос о разрешимости и решении сравнения $a^{n}(x) \equiv b(x)(\bmod F(x))$ над конечным полем для произвольного многочлена $F(x)$. В случае, если $F(x)$ является степенью неприводимого многочлена, приведен алгоритм подъема решений, то есть вопрос о решении сравнения

$$
a^{n}(x) \equiv b(x) \quad\left(\bmod f^{\alpha}(x)\right)
$$

сводится к вопросу о решении сравнения $a^{n}(x) \equiv b(x)(\bmod f(x))$. Для данного случая получены необходимые и достаточные условия разрешимости показательного сравнения. Если $F(x)$ не является степенью неприводимого многочлена, то решение сравнения по-прежнему сводится к решению сравнений вида $a^{n}(x) \equiv b(x)$ $\left(\bmod f_{i}(x)\right)$, а вопрос о проверке разрешимости сводится к проверке разрешимости сравнений вида

$$
a^{n}(x) \equiv b(x) \quad\left(\bmod f_{i}(x) f_{j}(x)\right),
$$

где $f_{i}(x)$ и $f_{j}(x)$ - неприводимые делители $F(x)$. Для модулей вида $f_{i}(x) f_{j}(x)$ результат получен в некоторых частных случаях.

Кроме того, описан конструктивный изоморфизм факторкольца многочленов $R=$ $G F\left(p^{m}\right)[x] /\left(f^{\alpha}(x)\right)$ и цепного кольца, представленного в виде $\bar{R}=G F\left(p^{r}\right)[x] /\left(x^{t}\right)$, благодаря чему полученные для многочленов результаты обобщаются на конечные цепные кольца простой характеристики. В частности, для цепных колец, представленных в виде $G F\left(p^{r}\right)[x] /\left(x^{t}\right)$, получены необходимые и достаточные условия разрешимости показательного сравнения.

\section{1. Введение}

Как известно, задача дискретного логарифмирования, то есть задача обращения функции $g^{n}$ в некоторой мультипликативной группе, является одной из ключевых в современной криптографии. На предположении о неполиномиальности сложности ее решения в некоторых группах основана стойкость многих криптографических схем.

Традиционно задача дискретного логарифмирования рассматривается в конечных полях или в группе точек эллиптической кривой. Однако эта задача вызывает интерес и применительно к другим алгебраическим объектам.

В [1] приведен алгоритм, который для произвольной конечной мультипликативной абелевой группы $G$ и элементов $g, d \in G, d \neq 1$, за $O(\sqrt{|\langle g\rangle|})$ умножений проверяет, 
принадлежит ли элемент $d$ циклической группе $\langle g\rangle$, порожденной элементом $g$, и в случае положительного ответа находит $\log _{g} d$. В данном алгоритме используется таблица, состоящая из $O(\sqrt{|\langle g\rangle|})$ пар элементов.

Заметим, что вопрос о принадлежности элемента циклической группе, порожденной другим элементом - это фактически вопрос о разрешимости соответствующей задачи дискретного логарифмирования. Естественно ожидать, что для решения этой задачи в какой-либо конкретной группе, структура которой нам известна, можно применить более эффективные алгоритмы. В частности, о некоторых алгоритмах дискретного логарифмирования в конечных полях можно прочитать в [2] (см. §5.4).

В [3] О. Н. Василенко рассматривал вопрос разрешимости задачи дискретного логарифмирования в факторкольце многочленов над простым конечным полем. Однако, в статье [3] были рассмотрены только два частных случая, содержащие довольно серьезные ограничения на порядки используемых элементов кольца.

В отличие от О. Н. Василенко, мы рассмотрим факторкольцо многочленов над произвольным конечным полем (а не только простым) и не будем вводить ограничений на порядки элементов. Мы будем изучать задачу дискретного логарифмирования в мультипликативной группе этого кольца.

Кроме того, одним из интересных алгебраических объектов являются коммутативные конечные цепные кольца простой характеристики. Структура данных колец подробно описана в [9] (см. гл. II, VI). Мы рассмотрим вопрос разрешимости и подъема решений задачи дискретного логарифмирования в таких кольцах.

Далее, если не сказано иного, предполагается, что все рассматриваемые элементы обратимы. Также мы будем подразумевать, что многочлен, по которому мы факторизуем, имеет старший коэффициент 1. Понятно, что это не ограничивает общность рассуждений: если старший коэффициент отличен от единицы, то мы всегда можем на него поделить (поскольку мы работаем над полем, любой ненулевой элемент обратим).

Раздел 2 посвящен рассмотрению задачи в кольце $R=G F(q)[x] /\left(f^{\alpha}(x)\right)$, где $q=p^{m}$, $p$ - простое число, $m \in \mathbf{N}, \alpha \in \mathbf{N} \backslash\{1\}$, а многочлен $f(x)$ неприводим над $G F(q)[x]$. Здесь мы полностью решаем задачу подъема решений показательного сравнения в указанном кольце, а также показываем, что алгоритм проверки разрешимости имеет сложность $O\left(\log ^{4}(|R|)\right)$.

В разделе 3 вопрос о разрешимости показательного сравнения в кольце $(G F(q)[x] /(F(x)))^{*}$, где многочлен $F(x)$ составной, сводится к вопросу разрешимости аналогичных сравнений по модулям вида $f_{i}(x) f_{j}(x)$, где $f_{i}(x)$ и $f_{j}(x)$ - неприводимые делители $F(x)$. В данном случае мы рассматриваем показательное сравнение в прямом произведении колец. Важно отметить, что из разрешимости сравнения в каждом из колец вовсе не следует существование решения в их прямом произведении, поскольку сами решения определены по некоторым, не взаимно простым, модулям. Однако для некоторых частных случаев возможно получить критерий разрешимости показательного сравнения в прямом произведении колец.

Далее мы рассмотрим общий случай конечных цепных колец. Как известно (см. теорему 3 главы VI в [9]), любое коммутативное кольцо характеристики $p$ изоморфно $G F\left(p^{r}\right)[x] /\left(x^{t}\right)$ для некоторого кольца Галуа $G F\left(p^{r}\right)$ и $t \in \mathbf{N}$. В разделе 4 будет построен изоморфизм колец $G F\left(p^{m}\right)[x] /\left(f^{\alpha}(x)\right)$ и $G F\left(p^{r}\right)[x] /\left(x^{t}\right)$, с помощью которого результаты раздела 2 будут легко обобщены на произвольные цепные кольца простой характеристики.

Автор выражает признательность своему научному руководителю М. А. Черепневу за помощь в изучении данной проблемы. 


\section{2. Разрешимость задачи дискретного логарифмирования и подъем решений показательного сравнения в кольце $\left(G F(q)[x] /\left(f^{\alpha}(x)\right)\right)^{*}$}

Рассмотрим факторкольцо многочленов $R=G F(q)[x] /\left(f^{\alpha}(x)\right)$, где $q=p^{m}, p$ - простое число, $\alpha \in \mathbf{N} \backslash\{1\}$, а многочлен $f(x)$ неприводим над $G F(q)[x], \operatorname{deg} f(x)=d$.

Теорема 1. Сравнение

$$
a(x) \equiv b(x) \quad\left(\bmod f^{\alpha}(x)\right)
$$

равносильно системе сравнений

$$
\begin{aligned}
a(x) & \equiv b(x) \quad(\bmod f(x)), \\
a^{q^{d}-1}(x) & \equiv b^{q^{d}-1}(x) \quad\left(\bmod f^{\alpha}(x)\right) .
\end{aligned}
$$

Доказательство. Заметим, что вместо степени $q^{d}-1$ можно выбрать также любое натуральное число, взаимно простое с $p$.

Любое решение сравнения, очевидно, удовлетворяет системе. Докажем утверждение в другую сторону. Из первого сравнения системы получаем, что

$$
a(x) \equiv b(x)+f^{l}(x) u(x), \quad f(x) \nmid u(x), \quad l \in \mathbf{N} .
$$

Если $l \geqslant \alpha$, то сравнение $a(x) \equiv b(x)\left(\bmod f^{\alpha}(x)\right)$ выполнено, то есть теорема доказана. Предположим, что $l<\alpha$. Возведем обе части сравнения в степень $q^{d}-1$. Воспользовавшись биномом Ньютона, получим, что

$$
a^{q^{d}-1}(x) \equiv b^{q^{d}-1}(x)+f^{l}(x)\left(\left(q^{d}-1\right) u(x)+f^{l}(x) T(x)\right) .
$$

С учетом второго уравнения системы получаем, что

$$
\begin{gathered}
f^{l}(x)\left(-u(x)+f^{l}(x) T(x)\right) \equiv 0 \quad\left(\bmod f^{\alpha}(x)\right), \\
u(x) \equiv f^{l}(x) T(x) \quad\left(\bmod f^{\alpha-l}(x)\right) .
\end{gathered}
$$

Получается, что $f(x) \mid u(x)$, что неверно по выбору $u(x)$. Значит, наше предположение неверно, следовательно, $l \geqslant \alpha$, и утверждение доказано.

Первое сравнение этой теоремы рассматривается в поле $G F(q)[x] /(f(x))$. Его разрешимость проверяется легко: достаточно, чтобы $\operatorname{ord}_{f(x)} b(x) \mid \operatorname{ord}_{f(x)} a(x)$, так как мультипликативная группа поля является циклической. Предполагается, что все разложения на множители нам известны, поэтому нахождение порядка элемента - простая задача (см. [4], гл. 1.4).

Приведем важную структурную теорему о кольце $R$ (см. теорему 6.6 .6 в [5]).

Теорема BS. Пусть $K=G F(q)[x] /(f(x))$. Тогда

$$
R^{*} \cong K^{*} \times(1+R f(x)), \quad|(1+R f(x))|=q^{d(\alpha-1)} .
$$

При этом максимальный порядок элемента группь $(1+R f(x))$, значение функиии Кармайкла, есть $\lambda(1+R f(x))=p^{k}$, где $k=\left\lceil\log _{p} \alpha\right\rceil$. 
В соответствии со структурной теоремой, $\operatorname{ord}_{R} a(x)=p^{\beta} \delta$, где $0 \leqslant \beta \leqslant k, \delta \mid q^{d}-1$. Если бы мы могли решить каждое из сравнений системы (2), то получили бы систему

$$
\begin{array}{ll}
n \equiv n_{1} & (\bmod \delta), \\
n \equiv n_{2} & \left(\bmod p^{\beta}\right) .
\end{array}
$$

Система (3) совместна, поскольку рассматриваемые модули взаимно просты. Следовательно, мы можем найти общее решение по китайской теореме об остатках (см. [6], гл. IV, §3). Кроме того, если оба сравнения системы (2) разрешимы, то и сравнение (1) разрешимо. Значит, и для проверки разрешимости, и для подъема решений нам нужно научиться решать второе сравнение системы (2).

Введем некоторые дополнительные обозначения.

Каждый элемент кольца $R$ можно однозначно представить в виде (см. лемму 5.32 в [2]):

$$
a(x) \equiv a_{0}(x)+a_{1}(x) f(x)+\ldots+a_{\alpha-1}(x) f^{\alpha-1}(x) \quad\left(\bmod f^{\alpha}(x)\right), \quad \operatorname{deg} a_{i}(x)<d .
$$

Пусть $K_{s}(a(x))=a_{s}(x)-s$-й коэффициент в разложении (4). Положим по определению $K_{\alpha}(a(x))=0$.

Пусть $u(x) \in R^{*}, \operatorname{ord}_{R} u(x)=p^{\beta}$. Из теоремы BS следует, что в этом случае $u(x) \equiv 1$ $(\bmod f(x))$. Значит, корректно определить следующую функцию

$$
D_{\alpha}(u(x))=\left\{\begin{array}{lll}
D: f^{D}(x) \| u(x)-1 & \text { при } u(x) \not \equiv 1 & \left(\bmod f^{\alpha}(x)\right), \\
\alpha, & \text { при } u(x) \equiv 1 & \left(\bmod f^{\alpha}(x)\right) .
\end{array}\right.
$$

Теперь мы можем сформулировать следующую теорему.

Теорема 2. Если $\operatorname{ord} u(x)=p^{\beta}$ и сравнение

$$
u^{n}(x) \equiv v(x) \quad\left(\bmod f^{\alpha}(x)\right)
$$

разрешимо относительно $n$, то

(1) $D_{\alpha}(v(x)) \geqslant D_{\alpha}(u(x))$;

(2) при $s=D_{\alpha}(u(x))$ сравнение

$$
y K_{S}(u(x)) \equiv K_{s}(v(x)) \quad(\bmod f(x))
$$

имеет иелочисленное решение.

В случае $u(x) \not \equiv 1\left(\bmod f^{\alpha}(x)\right)$, если $n$-решение сравнения (5), $y$-решение сравнения (6), $\operatorname{mo} n \equiv y(\bmod p)$.

Доказательство. Если $u(x) \equiv 1\left(\bmod f^{\alpha}(x)\right)$, то

$$
s=D_{\alpha}(u(x))=\alpha, \quad K_{s}(u(x))=K_{\alpha}(u(x))=0 .
$$

Если сравнение (5) разрешимо, то $v \equiv 1\left(\bmod f^{\alpha}(x)\right)$, и, следовательно, условия 1 и 2 теоремы выполнены.

Пусть далее $u(x) \not \equiv 1\left(\bmod f^{\alpha}(x)\right)$. Многочлен $u(x)$ представим в виде

$$
u(x) \equiv 1+u_{s}(x) f^{s}(x)+\ldots+u_{\alpha-1}(x) f^{\alpha-1}(x) \quad\left(\bmod f^{\alpha}(x)\right) .
$$


По биному Ньютона,

$$
u^{n}(x) \equiv 1+n u_{s}(x) f^{s}(x)+T(x) f^{s+1}(x) \quad\left(\bmod f^{\alpha}(x)\right) .
$$

Очевидно, что

$$
D_{\alpha}(v(x))=D_{\alpha}\left(u^{n}(x)\right) \geqslant D_{\alpha}(u(x))
$$

(равенство достигается тогда и только тогда, когда $p \nmid n)$ и

$$
K_{s}(v(x))=K_{s}\left(u^{n}(x)\right)=n u_{s}(x)=n K_{s}(u(x)) \quad(\bmod f(x)) .
$$

Таким образом, доказаны условия 1 и 2 теоремы.

Согласно определению, если $u(x) \not \equiv 1\left(\bmod f^{\alpha}(x)\right)$, то $K_{S}(u(x)) \neq 0$, поэтому сравнение (6) решается в поле $G F(q)[x] / f(x)=G F\left(q^{d}\right)$. Если $n, y \in Z$ и $n=y$ в $G F(q)$, то $n \equiv y(\bmod p)$. Теорема 2 доказана.

Данная теорема задает только необходимые условия для разрешимости сравнения (6), однако с ее помощью нетрудно построить алгоритм, проверяющий, имеет ли сравнение (1) решение, и, более того, находящий это решение по модулю $p^{\beta}$ в случае разрешимости.

Алгоритм. Входные данные: $q=p^{m}, f(x), \alpha, a(x) \not \equiv 1\left(\bmod f^{\alpha}(x)\right), b(x)$, разложение на простые множители числа $q^{d}-1$.

Задача: проверить, разрешимо ли сравнение (1); в случае разрешимости найти $\tilde{n}_{\beta} \in Z$ такое, что для решения $n$ сравнения (1) справедливо сравнение

$$
n \equiv \tilde{n}_{\beta} \quad\left(\bmod p^{\beta}\right),
$$

где $p^{\beta} \| \operatorname{ord} a(x)$.

Шаг 1. Найти ord $a(x)$.

Проверить, что $b^{\operatorname{ord} a(x)}(x) \equiv 1(\bmod f(x))$. Если не выполнено, то выход из алгоритма с отрицательным ответом.

Шаг 2. Найти $\beta: p^{\beta} \| \operatorname{ord} a(x)$.

$$
\begin{aligned}
& \tilde{a}(x)=a^{q^{d}-1}(x) \quad\left(\bmod f^{\alpha}(x)\right), \\
& \tilde{b}(x)=b^{q^{d}-1}(x) \quad\left(\bmod f^{\alpha}(x)\right), \quad i=0 .
\end{aligned}
$$

Шаг 3. Вычислить $s=D_{\alpha}(\tilde{a}(x))$.

Если $D_{\alpha}(\tilde{b}(x))<s$, то выход из алгоритма с отрицательным ответом.

Шаг 4. Решить линейное сравнение

$$
y K_{s}(\tilde{a}(x)) \equiv K_{s}(\tilde{b}(x)) \quad(\bmod f(x)) .
$$

Если его решение $y \notin Z$, то выход из алгоритма с отрицательным ответом; в противном случае $n_{i}=y, 0 \leqslant y<p, i=i+1$. 
Шаг 5. Если $i=\beta$, то $\tilde{n}_{\beta} \equiv n_{0}+n_{1} p+\ldots+n_{\beta-1} p^{\beta-1}\left(\bmod p^{\beta}\right)$, идти на шаг 6 .

В противном случае

$$
\begin{aligned}
& \tilde{b}(x)=\tilde{b}(x) \tilde{a}^{-n_{i-1}}(x) \quad\left(\bmod f^{\alpha}(x)\right), \\
& \tilde{a}(x)=\tilde{a}^{p}(x) \quad\left(\bmod f^{\alpha}(x)\right),
\end{aligned}
$$

идти на шаг 3.

Шаг 6. Проверить, что $a^{\left(q^{d}-1\right) \tilde{n}_{\beta}}(x) \equiv b^{q^{d}-1}(x)\left(\bmod f^{\alpha}(x)\right)$.

Если не выполнено, то выход из алгоритма с отрицательным ответом.

В противном случае выход с положительным ответом. Возврат $\tilde{n}_{\beta}$.

Теорема 3. Данный алгоритм проверяет разрешимость за $O\left(\log ^{4}(|R|)\right)$ операичй над полем $G F(q)$.

Доказательство. Вначале убедимся в корректности работы алгоритма.

Как уже говорилось выше, для разрешимости сравнения (1) нам необходимо и достаточно, чтобы были разрешимы оба сравнения системы (2). На шаге 1 мы проверяем разрешимость первого сравнения системы.

Далее, очевидно, что требуемое $\bar{n}_{\beta}\left(\bmod p^{\beta}\right)$ должно быть решением второго сравнения. На шагах 3-5 мы работаем со сравнением

$$
\tilde{a}^{n}(x) \equiv \tilde{b}(x) \quad\left(\bmod f^{\alpha}(x)\right)
$$

где $\tilde{a}(x) \equiv a^{\left(q^{d}-1\right) p^{i}}(x)\left(\bmod f^{\alpha}(x)\right), \operatorname{ord} \tilde{a}=p^{\beta-i}$.

На шагах 3 и 4 проверяются соответственно условия 1 и 2 теоремы 2 для сравнения (7). Обозначим $n^{(i)}$ решение данного сравнения. Тогда после шага 4 получаем, что $n^{(i)} \equiv n_{i-1}$ $(\bmod p)$.

Если $i<\beta-1$, то $n^{(i)} \equiv n_{i-1}+n_{i} p\left(\bmod p^{2}\right)$. Подставляя это выражение в сравнение (7), получаем, что

$$
\tilde{a}^{p n_{i}}(x) \equiv \tilde{b}(x) \tilde{a}^{-n_{i-1}}(x) \quad\left(\bmod f^{\alpha}(x)\right),
$$

что и объясняет шаг 5.

Если же $i=\beta-1$, то в конце шага 4 мы увеличим $i$ на 1 , на шаге 5 вычислим $\tilde{n}_{\beta}$ и перейдем на шаг 6. Если второе сравнение системы (2) разрешимо, то мы нашли его решение (в соответствии с теоремой 2), это и проверяется на шаге 6.

Итак, алгоритм работает корректно. Оценим время работы.

На шаге 1 необходимо найти мультипликативный порядок элемента. Это делается за $O\left(\log q^{d \alpha}\right)$ возведений в степень в $R$. Каждая степень требует $O\left(q^{d \alpha}\right)$, каждое возведение в степень требует $O\left(\log q^{d \alpha}\right)$ умножений многочленов над полем и делений с остатком. Умножение двух многочленов, степени которых не превосходят $d \alpha$, обычным алгоритмом требует $O\left((d \alpha)^{2}\right)$ операций над полем, деление с остатком требует столько же (см. [1], теорема 6.2.2). Итого, шаг требует $O\left(\log q^{d \alpha} \log q^{d \alpha} 2(d \alpha)^{2}\right)=O\left(\log ^{4}\left(q^{d \alpha}\right)\right)=O\left(\log ^{4}(|R|)\right)$ операций.

Шаги 2-5 повторяются $\beta$ раз.

На каждом шаге необходимо вычислить $K_{s}(\cdot)$, на что требуется не более чем $\alpha$ делений с остатком, а затем решить линейное сравнение, то есть, фактически, применить расширенный алгоритм Евклида. В соответствии с [1] (теоремы 6.2.2-6.2.4), деление с остатком потребует $O\left((d \alpha)^{2}\right)$, и расширенный алгоритм Евклида $O\left(d^{2}\right)$ шагов (так как 
многочлены имеют степени, не большие $d$ ). Итого, сложность шагов 2-5 составляет $O\left(\beta\left(\alpha 2(d \alpha)^{2}\right)=O\left(d^{2} \alpha^{4}\right)\right.$ операций.

На шаге 6, возведение в степень требует $O\left(\log \left(\left(q^{d}-1\right) \tilde{n}_{\beta}\right)\right)=O(d \alpha)$ умножений многочленов над полем и делений с остатком, то есть $O\left(d \alpha\left((d \alpha)^{2}\right)\right)=O\left(\log ^{3}(|R|)\right)$ операций над полем.

Итого, потребуется $O\left(\log ^{4}(|R|)\right)$ операций над полем $G F(q)$.

Заметим, что проверка условия 1 теоремы 2 на каждом шаге действительно необходима. Рассмотрим пример, в котором при $i=0$ это условие выполнено, а при $i=1$ нет.

Пример 1. Рассмотрим кольцо $G F(3)[x] /\left(\left(x^{2}+1\right)^{4}\right)$ и в этом кольце показательное сравнение

$$
\left(x^{4}\right)^{n} \equiv x^{4}+x^{2}+1 .
$$

Заметим, что

$$
\begin{aligned}
x^{4} & =\left(x^{2}+1\right)^{2}+\left(x^{2}+1\right)+1, \\
x^{4}+x^{2}+1 & =\left(x^{2}+1\right)^{2}+2\left(x^{2}+1\right)+1 .
\end{aligned}
$$

Тогда при $i=0$ получим, что

$$
K_{1}\left(x^{4}\right)=1, \quad K_{1}\left(x^{4}+x^{2}+1\right)=2, \quad n_{0}=2 .
$$

После этого, записав $n=2+3 n_{1}$, получим сравнение

$$
\left(x^{6}+2\right)^{n_{1}} \equiv x^{6}+x^{4}+2 x^{2}
$$

(промежуточные вычисления опускаем). Поскольку

$$
\begin{aligned}
x^{6}+2 & =\left(x^{2}+1\right)^{3}+1, \\
x^{6}+x^{4}+2 x^{2} & =\left(x^{2}+1\right)^{3}+\left(x^{2}+1\right)^{2}+1,
\end{aligned}
$$

условие 1 теоремы не выполняется, а следовательно, сравнение неразрешимо.

Кроме того, не лишним является и шаг 6. Возможна ситуация, при которой мы проверим условия 1 и 2 теоремы, но полученное $n$ не будет решением исходного сравнения.

Пример 2. Рассмотрим опять кольцо $G F(3)[x] /\left(\left(x^{2}+1\right)^{4}\right)$ и в этом кольце показательное сравнение

$$
\left(x^{4}\right)^{n} \equiv 2 x^{2}
$$

Заметим, что

$$
\begin{aligned}
x^{4} & =\left(x^{2}+1\right)^{2}+\left(x^{2}+1\right)+1, \\
2 x^{2} & =2\left(x^{2}+1\right)+1 .
\end{aligned}
$$

На первом шаге получим $n_{0}=2$, следовательно $n=2+3 n_{1}$. На следующем шаге получится сравнение $\left(x^{6}+2\right)^{n_{1}} \equiv x^{6}+2$, из которого получаем, что $n_{1}=1$. Порядок $x^{4}$ равен 9, следовательно мы должны выйти из цикла и вычислить $n$. Получаем, что $n=5$. Проверяем:

$$
\left(x^{2}\right)^{5} \equiv x^{10} \equiv x^{6}+2 x^{4}+x^{2}+1 \not \equiv 2 x^{2} .
$$

Следовательно, сравнение неразрешимо. 
Из теорем 1 и 2 легко вытекает следующая теорема.

Теорема 4. Если $\operatorname{ord}_{R} a(x)=p^{\beta} \delta$, где $0 \leqslant \beta \leqslant k, \delta \mid q^{d}-1$ и сравнение (1) разрешимо, то оно равносильно системе сравнений

$$
\begin{aligned}
a^{n}(x) & \equiv b(x) \quad(\bmod f(x)), \\
n_{i} K_{s}\left(a^{\left(q^{d}-1\right) p^{i}}(x)\right) & \equiv K_{s}\left(b^{q^{d}-1}(x) a^{-\left(q^{d}-1\right)\left(n_{0}+n_{1} p+\ldots+n_{i-1} p^{i-1}\right)}(x)\right) \quad(\bmod f(x)), \\
n & \equiv n_{0}+n_{1} p+\ldots+n_{\beta-1} p^{\beta-1} \quad\left(\bmod p^{\beta}\right) .
\end{aligned}
$$

Заметим, что в системе (8) присутствует $\beta+2$ сравнений. Проверка разрешимости сравнения (1), а также решение всех сравнений системы, кроме первого, осуществляется с помощью алгоритма, описанного выше. Таким образом, решение показательного сравнения из поля $G F(q)[x] / f(x)$ поднимается в кольцо $R$.

Похожая теорема, описывающая подъем решений показательного сравнения в кольце целых алгебраических чисел, доказана И. А. Поповяном в [7]. Вместо функции $K_{s}(\cdot)$ там использовались логарифм Артина-Хассе и $p$-адический логарифм.

\section{3. Разрешимость задачи дискретного логарифмирования в кольце $(G F(q)[x] /(F(x)))^{*}$ для произвольного многочлена $F(x)$}

Пусть $F(x)=f_{1}^{\alpha_{1}}(x) \ldots f_{s}^{\alpha_{s}}(x)$, где $s>1$ и все $f_{i}(x)$ неприводимы в $G F\left(p^{m}\right)[x]$. Тогда сравнение

$$
a^{n}(x) \equiv b(x) \quad(\bmod F(x))
$$

равносильно системе сравнений

$$
\begin{gathered}
a^{n}(x) \equiv b(x) \quad\left(\bmod f_{1}^{\alpha_{1}}(x)\right), \\
\cdots \\
a^{n}(x) \equiv b(x) \quad\left(\bmod f_{s}^{\alpha_{s}}(x)\right) .
\end{gathered}
$$

Таким образом, решение сравнения (9) сводится к решению аналогичных сравнений по модулям $f_{i}^{\alpha_{i}}(x)$. Следует обратить внимание на то, что для разрешимости сравнения (9) недостаточно разрешимости каждого из сравнений системы (10). Действительно, пусть все эти сравнения разрешимы и $n_{i}-$ решение $i$-го сравнения, $i=1, \ldots, s$. Пусть $\delta_{i}-$ мультипликативный порядок элемента $a(x)$ в кольце $G F\left(p^{m}\right)[x] /\left(f_{i}^{\alpha_{i}}(x)\right)$. Тогда система (10) равносильна системе

$$
\begin{aligned}
& n \equiv n_{1} \quad\left(\bmod \delta_{1}\right), \\
& \cdots \\
& n \equiv n_{s} \quad\left(\bmod \delta_{s}\right)
\end{aligned}
$$

Система (11) разрешима тогда и только тогда, когда

$$
n_{i} \equiv n_{j} \quad\left(\bmod \left(\delta_{i}, \delta_{j}\right)\right)
$$


для всех пар $i \neq j$ (см. [6], вопросы к гл. 4). В общем случае $n_{i}$ неизвестны, поскольку их нахождение сводится к дискретному логарифмированию в поле (по теореме 4), а это предположительно трудная задача. Возникает вопрос о том, как проверять разрешимость сравнения (9), не решая задачу дискретного логарифмирования.

Введем дополнительные обозначения. Пусть $\delta_{i}=p^{\beta_{i}} \delta_{i}^{\prime}$, где $\delta_{i}^{\prime} \mid\left(p^{m d_{i}}-1\right)$, $d_{i}=\operatorname{deg} f_{i}(x)$. Пусть $n_{i} \equiv \tilde{n}_{i}\left(\bmod p^{\beta_{i}}\right)$. Заметим, что $\tilde{n}_{i}$ можно вычислить с помощью алгоритма, приведенного в разделе 2 .

Теорема 5. Сравнение

$$
a^{n}(x) \equiv b(x) \quad\left(\bmod f_{1}^{\alpha_{1}}(x) \ldots f_{s}^{\alpha_{s}}(x)\right)
$$

разрешимо тогда и только тогда, когда выполнены следующие условия:

(1) для всех $i$ сравнение $a^{n}(x) \equiv b(x)\left(\bmod f_{i}^{\alpha_{i}}(x)\right)$ разрешимо;

(2) для всех $i \neq j$ сравнение $a^{n}(x) \equiv b(x)\left(\bmod f_{i}(x) f_{j}(x)\right)$ разрешимо;

(3) $\tilde{n}_{i} \equiv \tilde{n}_{j}\left(\bmod p^{\min \left(\beta_{i}, \beta_{j}\right)}\right)$.

Доказательство. Необходимость условий 1-3 доказывается легко. Действительно, если сравнение (9) выполнено по модулю $F(x)$, то оно выполнено и по модулям $f_{i}^{\alpha_{i}}(x)$, $f_{i}(x) f_{j}(x)$, поэтому выполнены условия 1 и 2. Далее, должна быть совместна система (11), а это означает, что выполнено условие (12). Из того, что $p^{\min \left(\beta_{i}, \beta_{j}\right)} \mid\left(\delta_{i}, \delta_{j}\right)$, получаем условие 3.

Докажем достаточность. Из условия 1 следует, что мы можем перейти к системе (11). Как уже говорилось, для разрешимости сравнения (9) должно быть выполнено условие (12). Поскольку $\left(\delta_{i}, \delta_{j}\right)=p^{\min \left(\beta_{i}, \beta_{j}\right)}\left(\delta_{i}^{\prime}, \delta_{j}^{\prime}\right)$, условие (12) можно заменить на условие

$$
\begin{array}{ll}
n_{i} \equiv n_{j} & \left(\bmod p^{\min \left(\beta_{i}, \beta_{j}\right)}\right), \\
n_{i} \equiv n_{j} & \left(\bmod \left(\delta_{i}^{\prime}, \delta_{j}^{\prime}\right)\right) .
\end{array}
$$

Первое сравнение данной системы выполнено в силу условия 3 теоремы.

Порядок $a(x)$ по модулям $f_{i}(x)$ и $f_{j}(x)$ равен соответственно $\delta_{i}^{\prime}$ и $\delta_{j}^{\prime}$. Следовательно, сравнение $a^{n}(x) \equiv b(x)\left(\bmod f_{i}(x) f_{j}(x)\right)$ равносильно системе

$$
\begin{array}{ll}
n \equiv n_{i} & \left(\bmod \delta_{i}^{\prime}\right), \\
n \equiv n_{j} & \left(\bmod \delta_{j}^{\prime}\right)
\end{array}
$$

Из условия 2 следует совместность этой системы, поэтому выполнено второе сравнение системы (13). Теорема доказана.

Из теоремы 5 легко получается следующее утверждение.

Следствие 1. Если сравнения $a^{n}(x) \equiv b(x)\left(\bmod f_{i}^{\alpha_{i}}(x)\right)$ разрешимы для всех $i$, то сравнение (9) равносильно системе сравнений

$$
\begin{aligned}
n & \equiv \tilde{n}_{i} \quad\left(\bmod p^{\beta_{i}}\right), \quad i=1, \ldots, s, \\
a^{n}(x) & \equiv b(x) \quad\left(\bmod f_{i}(x) f_{j}(x)\right), \quad i \neq j, \quad i, j=1, \ldots, s .
\end{aligned}
$$


Условия 1 и 3 теоремы 5 легко проверяются. Осталось научиться проверять условие 2. Пусть задано сравнение

$$
a^{n}(x) \equiv b(x) \quad(\bmod f(x) g(x))
$$

где $f(x)$ и $g(x)$ - различные неприводимые многочлены в $G F\left(p^{m}\right)[x]$. Обозначим $\delta_{1}$ и $\delta_{2}$ порядки многочлена $a(x)$ в факторкольцах $G F\left(p^{m}\right)[x] /(f(x))$ и $G F\left(p^{m}\right)[x] /(g(x))$ соответственно.

Лемма 1. Если $\left(\delta_{1}, \delta_{2}\right)=1$, то сравнение (15) разрешимо тогда и только тогда, когда разрешимы аналогичные сравнения по модулям $f(x)$ и $g(x)$.

Эта лемма следует из предыдущих рассуждений.

Пример 3. Пусть $p=2, d_{1}=\operatorname{deg} f(x), d_{2}=\operatorname{deg} g(x), m=1,\left(d_{1}, d_{2}\right)=1$. В этом случае максимальный возможный порядок элемента в кольце $G F(p)[x] /(f(x))$ равен $2^{d_{1}}-1$, а в кольце $G F(p)[x] /(g(x))$ равен $2^{d_{2}}-1$. При этом

$$
\left(2^{d_{1}}-1,2^{d_{2}}-1\right)=2^{\left(d_{1}, d_{2}\right)}-1=1,
$$

то есть условие леммы 1 выполнено для всех обратимых элементов.

Пусть $f(x)$ - неприводимый многочлен положительной степени со старшим коэффициентом 1. Тогда в кольце многочленов над конечным полем нечетной характеристики можно рассмотреть обобщенный символ Лежандра (см. [5], гл. 6.7), определяемый следующим образом:

$$
\left(\frac{g(x)}{f(x)}\right)= \begin{cases}1, & \text { если }(f(x), g(x))=1 \text { и } g(x) \text { - квадрат по модулю } f(x) ; \\ -1, & \text { если }(f(x), g(x))=1 \text { и } g(x) \text { - не квадрат по модулю } f(x) \\ 0 & \text { в противном случае. }\end{cases}
$$

При этом, если $g(x) \in G F(q)[x] /(f(x))$, то

$$
\left(\frac{g(x)}{f(x)}\right)=(g(x))^{\left(q^{d}-1\right) / 2} .
$$

Пусть $F(x)=f_{1}(x) \ldots f_{r}(x)$, где $f_{i}(x)$ - неприводимые многочлены со старшими коэффициентами 1. Обобщенный символ Якоби определяется равенством

$$
\left(\frac{g(x)}{F(x)}\right)=\prod_{i=0}^{r}\left(\frac{g(x)}{f_{i}(x)}\right) .
$$

Если $F(x), G(x) \in G F(q)[x]$ - многочлены со старшим коэффициентом 1 , то выполнен аналог квадратичного закона взаимности Гаусса:

$$
\left(\frac{G(x)}{F(x)}\right)=(-1)^{(q-1)(\operatorname{deg} F(x))(\operatorname{deg} G(x)) / 2}\left(\frac{F(x)}{G(x)}\right) .
$$

Алгоритм вычисления обобщенного символа Якоби для произвольных многочленов $F(x)$ и $G(x)$ описан в [5]. В этом алгоритме используется закон обращения (17), а также квадратичный характер $\chi(c)=c^{(q-1) / 2}$ в поле $G F(q)$. 
Лемма 2. Если $p-$ нечетное число, $\left(\delta_{1}, \delta_{2}\right)=2,4 \nmid p^{m d_{1}}-1,4 \nmid p^{m d_{2}}-1$, то сравнение (15) разрешимо тогда и только тогда, когда разрешимы аналогичные сравнения по модулям $f(x)$ и $g(x)$ и $\left(\frac{b(x)}{f(x) g(x)}\right)=1$.

Доказательство. По условию $\left(\delta_{1}, \delta_{2}\right)=2,4 \nmid p^{m d_{i}}-1$, поэтому из равенства (16) следует, что $a(x)$ является квадратичным невычетом по обоим модулям $f(x)$ и $g(x)$.

Необходимость того, что сравнения по модулям $f(x)$ и $g(x)$ разрешимы, очевидна. Пусть $n_{1}$ и $n_{2}$ - решения. Тогда для разрешимости исходного сравнения необходимо и достаточно, чтобы $n_{1} \equiv n_{2}(\bmod 2)($ см. $(13),(14))$, то есть $n_{1}$ и $n_{2}$ имеют одинаковую четность, а $b(x)$ является либо квадратичным вычетом, либо невычетом по обоим модулям одновременно, то есть

$$
\left(\frac{b(x)}{f(x)}\right)=\left(\frac{b(x)}{g(x)}\right) .
$$

По свойствам обобщенного символа Якоби получаем, что

$$
\left(\frac{b(x)}{f(x) g(x)}\right)=1 .
$$

Пример 4. Пусть $p=3, d_{1}=\operatorname{deg} f(x), d_{2}=\operatorname{deg} g(x), m=1,\left(d_{1}, d_{2}\right)=1, d_{1}, d_{2}-$ нечетные числа. В этом случае $4 \nmid 3^{d_{i}}-1=2\left(3^{d_{i}-1}+\ldots+3+1\right)$. Кроме того,

$$
\left(3^{d_{1}}-1,3^{d_{2}}-1\right)=3^{\left(d_{1}, d_{2}\right)}-1=2,
$$

а значит, для любого обратимого элемента $a(x)$ выполнены условия леммы 1 или леммы 2, и разрешимость сравнения (15) легко проверить.

\section{4. О цепных кольцах характеристики $p$}

Заметим, что все нетривиальные идеалы в кольце $R=G F\left(p^{m}\right)[x] /\left(f^{\alpha}(x)\right)$ имеют вид $f^{c}(x) R$. Действительно, кольцо многочленов от одной переменной над полем является кольцом главных идеалов (см. [8], раздел 17). Факторкольцо кольца главных идеалов является кольцом главных идеалов (см. [9], гл. 1, пример 8), следовательно, $R$ - кольцо главных идеалов. Легко проверить, что идеал, порожденный обратимым элементом, совпадает с кольцом $R$. Значит, все нетривиальные идеалы порождаются обратимыми элементами, то есть элементами, делящимися на $f(x)$.

Следовательно, для любых нетривиальных идеалов $I$ и $J$ выполнено либо включение $I \subset J$, либо $J \subset I$, то есть кольцо $R$ является цепным (см. [8], гл. II, теорема 6). Любое коммутативное конечное цепное кольцо характеристики $p$ изоморфно $\bar{R}=G F\left(p^{r}\right)[x] /\left(x^{t}\right)$ для некоторых $t, r \in \mathbf{N}$ (см. [8], гл. VI, следствие теоремы 3). Нетрудно проверить, что $r=m d, t=\alpha$, и построить изоморфизм $R \cong \bar{R}$ в явном виде.

В кольце $R$ существует элемент $u(x)$ порядка $p^{m d}-1$ (по теореме $\mathrm{BS}$ ).

Кольцо $R$ является локальным кольцом (см. [8], гл. II, теорема 6) характеристики $p$, следовательно,

$$
\left\{0, u(x), u^{2}(x), \ldots, u^{p^{m d}-1}(x)\right\} \cong G F\left(p^{m d}\right)
$$

(см. [8], гл. VI, лемма 2). Пусть $\bar{u} \in \bar{R}-$ образ $u(x)$ при этом изоморфизме.

Любой элемент кольца $R$ однозначно представляется в виде

$$
a(x) \equiv a_{0}(x)+a_{1}(x) f(x)+\ldots+a_{\alpha-1}(x) f^{\alpha-1}(x) \quad\left(\bmod f^{\alpha}(x)\right),
$$


где $a_{i}(x)=0$ или $a_{i}(x) \equiv u^{c_{i}}(x)\left(\bmod f^{\alpha}(x)\right), c_{i} \in\left\{1, \ldots, p^{m d}-1\right\}$.

Подобное разложение строится по индукции. Вначале находятся

$$
a_{i}^{\prime}(x) \equiv \frac{a(x)-a_{0}^{\prime}(x)-a_{1}^{\prime}(x) f(x)-\ldots-a_{i-1}^{\prime}(x) f^{i-1}(x)}{f^{i}(x)} \quad(\bmod f(x)),
$$

после чего для каждого $i$, если $a_{i}^{\prime}(x) \neq 0$, в поле $G F(q)[x] / f(x)$ ищется единственное $c_{i}$ такое, что $a_{i}^{\prime}(x) \equiv u^{c_{i}}(x)(\bmod f(x))$, а затем вычисляются $a_{i}(x)$. Заметим, что конструктивное построение разложения (18) является сложным, поскольку требует решения задачи дискретного логарифмирования в поле.

Рассмотрим отображение $\varphi: R \rightarrow \bar{R}$, действующее следующим образом: элемент $a(x) \in R$, заданный представлением (18) переходит в

$$
\bar{a}(x) \equiv \bar{a}_{0}+\bar{a}_{1} x+\ldots+\bar{a}_{\alpha-1} x^{\alpha-1} \quad\left(\bmod x^{\alpha}\right),
$$

где $\bar{a}_{i}=0$, если $a_{i}(x) \equiv 0(\bmod f(x))$ и $\bar{a}_{i}=\bar{u}^{c_{i}}$ в противном случае.

Теорема 6. Отображение $\varphi(\cdot)$, описанное формулами (19), является изоморфизмом.

Доказательство. Рассмотрим $a(x), b(x) \in R$ и $T(x)=a(x)+b(x)$, а также их образы $\bar{a}(x), \bar{b}(x), \bar{T}(x) \in \bar{R}$. Докажем, что $\bar{T}(x)=\bar{a}(x)+\bar{b}(x)$. Достаточно доказать, что

$$
\bar{T}_{i}=\bar{a}_{i}+\bar{b}_{i} .
$$

Рассмотрим $i$-е коэффициенты в разложении (18). Если $a_{i}(x)=0$ или $b_{i}(x)=0$, то, очевидно, (20) выполнено. В противном случае (20) следует из выбора $\bar{u}$.

Аналогичным образом доказывается, что $\varphi$ сохраняет произведение, а следовательно является изоморфизмом.

С учетом построенного изоморфизма нетрудно переформулировать теоремы, доказанные в разделе 2 , для кольца $\bar{R}$. Заметим, что функция $K_{S}(\cdot)$ в данном случае будет просто $s$-м коэффициентом многочлена.

Таким образом, если для некоторого цепного кольца построен эффективный изоморфизм в $\bar{R}$, мы можем, с учетом раздела 2 , решать в нем задачу подъема решений и пользоваться критерием разрешимости показательного сравнения из раздела 3.

Кроме того, можно использовать другой конструктивный изоморфизм этих колец и модифицировать алгоритм проверки разрешимости сравнения, описанный в разделе 2 . Пусть $t$ - корень многочлена $f(x)$ над $G F\left(q^{d}\right)$. Тогда изоморфизм ставит в соответствие элементу $a(x) \in R$ элемент $a(x+t) \in \bar{R}$.

Следовательно, мы можем модифицировать наш алгоритм: вначале вычислить $\bar{a}(x)=a(x+t) \in \bar{R}$ и $\bar{b}(x)=b(x+t) \in \bar{R}$, а затем выполнить шаги 1-6 алгоритма для $\bar{a}(x)$ и $\bar{b}(x)$ в кольце $\bar{R}$.

Рассмотрим, как изменится сложность алгоритма в случае, когда $f(x)=x$. Понятно, что в этом случае мы избавляемся от всех делений с остатком. Кроме того, степени всех многочленов не превосходят $\alpha$ (а ранее $d \alpha)$.

Таким образом, на шаге 1 выполняется $\left.O\left(\log q^{d \alpha} \log q^{d \alpha}(\alpha)^{2}\right)\right)=O\left(\alpha^{2} \log ^{2}(|\bar{R}|)\right)$ операций.

Сложность шагов 2-5 составляет $O(\beta \alpha)=O\left(\alpha^{2}\right)$ операций.

Сложность шага 6 есть $O\left(d \alpha \alpha^{2}\right)=O\left(\alpha^{2} \log (|\bar{R}|)\right)$.

Итого потребуется $O\left(\alpha^{2} \log ^{2}(|\bar{R}|)\right)$ операций над полем $G F\left(q^{d}\right)$.

Поскольку операция умножения над полем $G F(q)$ имеет сложность $O\left(\log ^{2} q\right)$ битовых операций, а над $G F\left(q^{d}\right)$ соответственно $O\left(\log ^{2} q^{d}\right)=O\left(d^{2} \log ^{2} q\right)$, порядок сложности 
алгоритмов получается одинаковым. На шагах 1 и 6 мы получаем в точности одинаковые значения.

Отметим, что шаги 1 и 6 можно выполнять в любом из колец, то есть возможно делать переход в кольцо $\bar{R}$ только для выполнения шагов $2-5$.

Второй алгоритм дает выигрыш на шагах 2-5. Это получается за счет упрощения нахождения $K_{S}$ (не требуется делить многочлены, просто выписываются коэффициенты).

Во втором алгоритме требуется еще нахождение элементов $\bar{a}(x)$ и $\bar{b}(x)$. Для этого потребуется вычисление $(x+t)^{i}$ для $i=1, \ldots, d \alpha$, то есть $d \alpha$ умножений многочлена 1 -й степени на многочлен $c_{i-1}(x) \equiv(x+t)^{i-1}\left(\bmod \left(x^{\alpha}\right)\right.$ степени $O(\alpha)$. Сложность будет равна $O\left(d \alpha^{2}\right)$ операций над $G F\left(q^{d}\right)$, то есть $O\left(d^{3} \alpha^{2} \log ^{2} q\right)$ битовых операций.

Итак, для выполнения шагов 2-5 в $R$ получаем сложность $O\left(d^{2} \alpha^{4} \log ^{2} q\right)$ битовых операций, а в случае кольца $\bar{R}$ (с учетом построения изоморфизма) получаем $O\left(\left(d^{3} \alpha^{2}+d^{2} \alpha^{2}\right) \log ^{2} q\right)$ битовых операций.

Таким образом, каждый из алгоритмов может иметь преимущества по скорости в зависимости от соотношения величин $\alpha^{2}$ и $d$.

\section{Список литературы}

1. Buchmann J., Jacobson M. J., Teske E., On some computational problems in finite abelian groups. Math. Comput. (1997) 66, №220, 1663-1687.

2. Василенко О. Н., Теоретико-числовые алгоритмы в криптографии. МЦНМО, Москва, 2003.

3. Василенко О. Н., О дискретном логарифмировании в некоторых группах. Вестник МГУ, Сер. 1: матем., механ. (2000), № 5, 53-55.

4. Cohen H., A course in computational algebraic number theory. Springer, Berlin, 1993.

5. Bach E., Shallit J., Algorithmic number theory, 1. MIT, Massachusetts, 1996.

6. Виноградов И. М., Основы теории чисел. Наука, Москва, 1972.

7. Поповян И. А., Подъем решений показательного сравнения. Математические заметки (2006) 80, №1, 76-86.

8. Ван дер Варден Б. Л., Алгебра. Наука, Москва, 1976.

9. Елизаров В. П., Конечные кольца. Гелиос АРВ, Москва, 2006.

Статья поступила 15.09.2009. 